DIVISION OF THE HUMANITIES AND SOCIAL SCIENCES

CALIFORNIA INSTITUTE OF TECHNOLOGY

PASADENA, CALIFORNIA 91125

HEDONISM VS. NIHILISM: NO ARBITRAGE AND TESTS OF URBAN ECONOMIC MODELS

Marcus Berliant

California Institute of Technology and

Washington University

Daniel P. McMillen

University of Illinois at Chicago

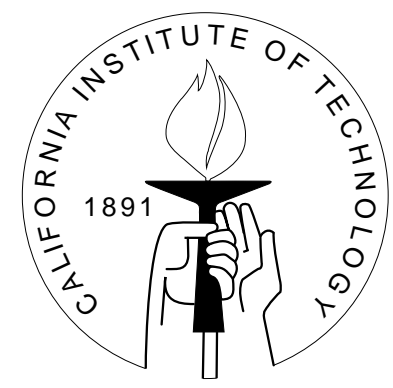

SOCIAL SCIENCE WORKING PAPER 1166

May 2003 


\title{
Hedonism vs. Nihilism: No Arbitrage and Tests of Urban Economic Models
}

\author{
Marcus Berliant Daniel P. McMillen
}

\begin{abstract}
We present two notions of "no arbitrage" in urban economic models and show that there is no model satisfying both. The standard hedonic housing model of urban economics and its generalizations are consistent with the first of these, but inconsistent with the second. We present a model consistent with the second notion of "no arbitrage" and a continuum of models consistent with neither notion that are observationally equivalent to the standard model, even if the utility function of consumers is known. They generate the same equilibrium allocations and values of bundles purchased in equilibrium, but the equilibrium price functions differ on bundles not purchased in equilibrium. Only one of these is the standard model. Thus, the available tests of the standard model cannot provide much evidence of its validity.
\end{abstract}

Keywords: Monocentric City; Hedonic Models; No Arbitrage

JEL classification numbers: R21, R13, D41, C21

*The authors thank participants at the real estate seminar of the Haas School of Business at the University of California, Berkeley. The authors also thank Jan Brueckner, Ed Coulson, John Nachbar, Bob Pollak, Paul Rothstein, Suzanne Scotchmer, Jacques Thisse, Nancy Wallace and particularly David Pines for helpful comments, but retain full responsibility for any errors. The first author gratefully acknowledges financial support from the American Philosophical Society. E-mail addresses: Marcus Berliant (berliant@wueconc.wustl.edu); Daniel P. McMillen (mcmillen@uic.edu) 


\section{Introduction}

The empirical tests of standard urban economic models involve their comparative statics as well as characterizations of equilibrium. The characterizations of equilibrium generally include conditions such as a downward sloping rent function as one moves away from a city center or central business district (CBD), increasing land consumption with distance from the CBD, as well as a "Muth-Mills" condition. The latter states that the change in land price as one moves farther from the CBD multiplied by land consumption at the base location is exactly equal to the change in commuting cost multiplied by $(-1)$. An analogous expression replacing "land" with "housing" can be formulated, depending on the commodities placed in the model.

Examples of work testing urban models include Mills (1969), Kau and Sirmans (1979), Yinger (1979), McMillen (1990) and McMillen (2003). Of particular importance to us is Coulson (1991), which is the origination of the ideas we present. ${ }^{1}$

Since we attempt here to cover large classes of urban economic models, we do not use assumptions on their primitives, such as utility functions and preferences, but rather we assume that general implications of equilibrium in the models hold. These assumptions are the ones usually considered to have empirical content. We do not need to restrict attention to the standard monocentric city model, though that model is included in the class we consider. Instead, we examine the structure of the empirical models constructed to test the implications of urban economic models; clearly, these are reduced form rather than structural models.

The first assumption we impose is that there are at least two locations and two house types. Second, we assume that in each location, there are no arbitrage possibilities at equilibrium. What this means is that the value of housing or land at a location is proportional to its quantity. That is, the cost of a house is its price times quantity. Finally, we impose the ceterus paribus condition used to derive the Muth-Mills condition and its variants: if we take a house and move it farther from the CBD, its price must go down by exactly the commuting cost (actually, the present discounted value of commuting cost over the infinite time horizon). Notice that we do not require that identical houses actually are built in equilibrium at different distances from the CBD, for this would contradict classical results for the monocentric city model, that

\footnotetext{
${ }^{1}$ See, in particular, pp. 300-301 of that paper. We noticed that conditions [3] and [4] seemed to be in contradiction, though they are derived from the same model.
} 
house or land consumption strictly increases with distance from the CBD. Rather, we only require that such houses be priced in equilibrium. In fact, the pricing of all possible houses in all locations is necessary even to have agent optimization problems well-defined.

From these three assumptions, we obtain a contradiction. We conclude that the common tests of the hedonic housing models actually contradict one of the no arbitrage conditions. Next we formulate an example to motivate our result.

Example 1 Suppose that the present discounted value of commuting cost is $\$ 2000$ per mile (this is in rough agreement with the empirical estimate of Coulson (1991, p. 304)). Suppose that a nice 2-bedroom house sells for \$100,000 at distance 1 mile from the CBD. So if this house were available 2 miles from the CBD, it would sell for \$98,000. Now consider a 3-bedroom house, and suppose that it would sell for \$150,000 at distance 1 mile from the CBD if it were available. Suppose that it actually sells for $\$ 148,000$ and is available 2 miles from the CBD (in equilibrium). Now let's calculate the per unit cost of housing, assuming that housing is priced proportionately. If a 2-bedroom house were given housing quantity 2, then the per unit cost of housing at distance 1 mile from the $C B D$ is $\$ 50,000$. If the 3-bedroom house were given housing quantity 3, then its unit cost is also \$50,000. Now let's look at the unit cost of housing at distance 2 miles from the CBD. The per unit cost of the 2-bedroom house is \$49,000. The per unit cost of the 3-bedroom house is \$49,333.33. This contradicts "no arbitrage" in the housing good. Independent of whether these houses are actually built in equilibrium, the hedonic price function must be well-defined for all possible houses in all locations.

In essence, the argument is that if commuting cost differences have to be spread over different numbers of units of housing, then there will be a violation of no arbitrage, since the unit cost of housing must be different for the different houses. The same argument would apply if we used land parcels instead of houses.

The components critical to this example are that (1) no arbitrage implies a linear housing price function at a given location, and (2) the Muth-Mills model implies changes in prices for identical houses across locations that depend only on commuting costs. In standard urban models, at equilibrium, houses are generally identical at a given location, and houses generally differ across locations. However, hedonic models require that all houses be priced at all 
locations, even when they are not actually present in equilibrium. Empirically, neighboring houses are often quite different and the same houses often are built at very different locations. No arbitrage requires that the hedonic price function be linear for houses in the same neighborhood. The Muth-Mills model implies that houses that are identical in all ways except location must differ in price by commuting cost multiplied by the difference in distance from the city center. A price schedule in which prices vary across locations independently of house characteristics is inconsistent with no arbitrage at each location.

After stating and proving this result, we shall examine equilibrium price systems that satisfy one of the two no arbitrage conditions, as well as some that satisfy neither. There is a continuum of price systems (or models) that yield the same equilibrium allocation (the one generated by the standard monocentric city model) and price the bundles purchased in equilibrium the same, but that disagree for bundles not purchased in equilibrium. Thus, all of these models are observationally equivalent, so the "tests" of the standard model cannot distinguish it from the rest of these models, even if the consumer wealth and utility function are known. We present an example based on quasi-linear utility. How bundles not purchased in equilibrium are priced is crucial for assessment, for instance, since in the real world, heterogeneous houses in the same neighborhood can be found.

The bottom line is that there is no price system or model that satisfies both no arbitrage conditions, and there is a continuum of models that are observationally equivalent to the standard model. There is one such model consistent with each of the two no arbitrage conditions, and a continuum of models consistent with neither.

It is important to relate our work to the general literature on hedonic pricing, such as Rosen (1974) or Mas-Colell (1975) and the many papers that followed them. The link is that location is one of many characteristics of a differentiated commodity called housing. The first and most obvious difference is that there is no location or commuting cost in the general hedonic model. Thus, there is no analog of the Muth-Mills condition in the general model, so a no arbitrage condition that applies across locations (or one dimension of differentiated goods) is lacking. Second, there are usually indivisibilities in the general literature, so complete repackaging of commodities is not allowed. No arbitrage at a given location or for a given type of good will not hold in general because the good cannot be completely repackaged when some characteristics are fixed or indivisible. On the other hand, in urban hedonic 
models, if the locational or differentiated good is land at a particular location, then repackaging is clearly possible since the quantity of land purchased is not fixed. If the locational good is housing differentiated by location, then there is the potential for a no repackaging assumption or an indivisibility. In urban economic theory, it has simply been traditionally assumed that the housing good is divisible, and this is the kind of model we analyze. Moreover, for our results below, we only require that two types (or packages) of houses be conceivable; we do not require that arbitrary linear combinations of a housing good be possible. In empirical urban economics, linearity of prices at the micro level has been used to identify submarkets in a small geographical region. So an assumption of no repackaging of commodities would seem not to be empirically relevant.

We begin with the notation and assumptions in Section 2. Section 3 of the paper simply makes Example 1 formal and general. Section 4 discusses the continuum of models that are observationally equivalent to the standard monocentric city model. Section 5 contains our conclusions.

\section{The Notation and Assumptions}

Our assumptions are not on primitives. This is done both to reduce the amount of notation as well as to cover classes of models that generate the same or similar testable implications. The following basic framework is typical of most urban models. We assume that location is represented by a variable $x \in$ $X \subseteq \Re_{+}$, the set of all possible locations. We assume that the CBD to which every consumer must commute is represented by $0 \in X$. Multiple subcenters would cause no problems for us, but we defer the discussion of extensions to Section 5. The housing good (or land) is represented by $h \in H \subseteq \Re_{+}$. We define $G$ to be an abstract set of other locational characteristics, and let $g \in G$ be a generic element of the set. (The set $G$ need not be a subset of a Euclidean space.) For example, suppose that there were certain characteristics that vary with location in equilibrium, such as neighborhoods, schools and local public goods in general. These need not be exogenous variables in the structural model. The value of $g$ could be exogenous (for example, if we took schools associated with a location as exogenous) or it could be the equilibrium value of some endogenous variables, like neighborhood income. Then the hedonic pricing function takes as its domain housing purchase, location, and the values

of these characteristics at the selected location. In other words, we write the 
hedonic pricing function as $P(h, x, g)$, where $P: H \times X \times G \rightarrow \Re$. It value is the total price of the specified house, not the unit cost. If there are no locational characteristics other than location itself, we can simply let $G$ be a singleton (constant across location). In fact, if one interprets $h$ as land consumption, one could also include in $g$ other housing characteristics, such as the number of bedrooms. Our analysis is robust to all of these variations and interpretations. In general, we will assume that $P$ is the equilibrium hedonic price function, since we shall be examining conditions that characterize equilibrium in urban models. This formulation of the hedonic price function is simple but general. We assume that commuting cost is given by $t \cdot x$ for a person living at $x \in X$, where $t>0$. This is also standard in the literature.

In hedonic models, it is necessary to have prices defined for all possible bundles of goods at each location. For example, this is common in the local public finance literature, where membership in every jurisdiction, whether it happens to be present in equilibrium or not, must be priced (see Ellickson, Grodal, Scotchmer and Zame (2000) for a recent incarnation of this feature). The reason, of course, is that without prices for some goods (or jurisdictions), the optimization problems of agents are not well-defined since they cannot compute the cost of each bundle. In our context, the implication is that $P(h, x, g)$ must be defined for each $x \in X$, each $h \in H$ and each $g \in G$. The theoretical models imply that all house types or lot sizes must be priced at all locations. The empirical hedonic models actually do price all house types at all locations. Of course, this is necessary in order to assess properties, one of the main applications of the model.

Axiom 1 : There are at least two distinct members of $X$ (called $x$ and $y$ ), at least two distinct members of $H$ (called $h$ and $i$ ), and at least one member of $G, g \in G$.

It shouldn't be too controversial to assume that there are at least two locations and two types of houses. Moreover, we assume that there is at least one common member of $G$ that is priced across these two locations and for these two housing types. As long as the hedonic price function $P$ is defined for the four bundles $P(h, x, g), P(i, x, g), P(h, y, g)$, and $P(i, y, g)$, how the hedonic price function $P$ is defined for other bundles is completely irrelevant. Without loss of generality, we take $H=\{h, i\}$, $X=\{x, y\}$, and $G=\{g\}$. For consistency below, we will often say " $\forall g \in G$ "; for our purposes, the reader can take this to mean "for the unique element of $G^{\prime \prime}$. 
Axiom 2 (No Arbitrage): At each location, the total cost of housing is linear in the quantity consumed. Formally, $\forall g \in G, \forall x \in X, \forall h \in H, P(h, x, g)=$ $h \cdot p(x, g)+c(g)$, where $p: X \times G \rightarrow \Re$ and $c: G \rightarrow \Re$.

The interpretation, of course, is that people can not make positive profits by simply trading in land or housing at a given location. The price function $p(x, g)$ is the type usually used in the urban economics literature. It represents the per unit price of housing with characteristics $g$ located distance $x$ from the $\mathrm{CBD}$. If one wants to dispense with this condition and use nonlinear pricing for $h$, then one can kiss the first welfare theorem good-bye; equilibrium allocations might not be Pareto optimal. The usual proof of the first welfare theorem relies heavily on linearity of prices. In the theoretical literature, it is typical that $c \equiv 0$. In the empirical literature, it is typical for $c$ to represent components of the regression other than terms and interaction terms involving $h$.

When $H$ is land rather than housing and $c \equiv 0$, the interpretation of Axiom 2 is particularly compelling. If we consider $h$ and $i$ to be vacant lots where one is twice the size of the other, then since the parcels can be split into pieces, no arbitrage says that agents cannot make a profit by breaking the parcels up or combining them. ${ }^{2} \quad$ Vacant lots need not be present in equilibrium; we only require that the hedonic price function prices them. We allow the generalization $c \neq 0$ to cover empirical work.

When $H$ is housing, since we only need to use the values at two points (at each location), this is really just agreement (among agents) about the units used to measure the housing good. Such an assumption is usually justified in the literature by a production function for housing that is locationindependent (often Cobb-Douglas in capital and land). This leads to a location-independent scale for measuring housing, though the price of housing can still be location-dependent due to the location-dependence of the price of land. In that case, lot size can either be embodied in $h$ through the production function and/or included in $g$.

One way to capture many of the empirical models in our framework is to make $h$ land and throw all of the housing characteristics into $g$. Then the houses we are comparing differ only in lot size and, once again, no arbitrage in land seems compelling.

Empirically, this is a semiparametric regression.

\footnotetext{
${ }^{2}$ As is standard in most of economics, we assume that consumers ignore feasibility constraints when solving their optimization problems, so they think it's possible to purchase vacant lots of any size at any location.
} 
Axiom 3 (Coulson-Muth-Mills): If we take a house and move it $d$ units farther from the $C B D$, then its cost goes down by the commuting cost multiplied by d. Formally, $\forall g \in G, \forall h \in H, \forall x, y \in X, P(h, x, g)=P(h, y, g)+t \cdot[y-x]$.

There are many remarks to be made about the last axiom. First, the intuition is clear and well-known. If we take two houses or vacant lots that are identical in every respect except distance from the $\mathrm{CBD}$, then the difference in price must be equal to the commuting cost. In terms of Coulson (1991), equation [3] of that paper is the differential version of our Axiom 3. In our notation, presuming that $P$ is differentiable in $x$, Coulson's equation [3] is

$$
\partial P(h, x, g) / \partial x=-t .
$$

This can be derived from Axiom 3 by letting $x$ tend to $y$ and using the definition of a derivative.

As Coulson notes, the argument for the standard Muth-Mills condition is a bit harder, since the ceterus paribus conditions are more complicated. That's because the equilibrium consumption of housing increases with distance from the CBD under the "normal" ${ }^{3}$ assumptions. Let $h(x)$ denote the equilibrium value of $h$ at $x$, the number of units of housing or land purchased in equilibrium at distance $x$ from the CBD. In terms of our notation, the standard differential version of the Muth-Mills condition is as follows.

$$
\partial p(x, g) / \partial x \cdot h(x)=-t
$$

It can be derived from the budget constraint and equal utility equilibrium condition of the standard model (see the usual texts Fujita (1989) or Mills and Hamilton (1994, chapter 6), or see Coulson (1991, equation [4a])), though its derivation can be a bit messy.

We prefer the discrete version since it simplifies our analysis and avoids the use of additional assumptions, such as differentiability of $P$. The discrete version is a bit more involved.

$$
\forall x, y \in X, P(h(y), y, g)+t \cdot y \leq P(h(y), x, g)+t \cdot x
$$

\footnotetext{
${ }^{3}$ That is, the usual assumptions plus normality of housing. Pun intended. See Fujita (1989).
} 
The intuition here is pretty much the same as the other intuitions we have advanced. Bear in mind that in these models, price is generally decreasing with distance from the $\mathrm{CBD}$ while housing consumption is generally increasing with distance from the CBD. If we look at the equilibrium housing profile across locations $h(y)$, then if we move farther from the CBD and look at the same house, the price decrease from moving farther from the CBD must not exceed the increase in commuting cost. If we move closer to the CBD and look at the same house, the price increase must be at least as large as the decrease in commuting cost. The reason this differs from the differential statement (2) above that uses only the equilibrium value of housing at a point is that in the discrete case, equilibrium housing consumption changes with a discrete change in location, and no arbitrage should prevent consumers from moving and buying the identical house in equilibrium. Evidently, we can obtain the differential version (2) by imposing Axiom 2, by using the inequalities (3), and by applying the definition of derivative by taking limits as $x$ approaches $y .{ }^{4}$ Notice that the difference between our inequality (3) and our Axiom 3 is that Axiom 3 uses equalities, while inequality (3) uses inequalities. In fact, Axiom 3 implies inequality (3), but not conversely.

So how can we obtain Axiom 3 from the discrete version of the Muth-Mills condition (as opposed to the Coulson condition)? Here it is useful to quote Ed Coulson (1991, p. 301). "Furthermore, in a hedonic regression where distance and other housing attributes are included on the right-hand side, the coefficient on location must be interpreted in precisely the ceterus paribus context noted for [3]." Our equation (1) is his equation [3] in our notation. In other words, when hedonic regressions are run to test urban models (or for other purposes, such as real estate assessment), equation (1) and thus Axiom 3 are implicitly assumed, since houses not priced by the Muth-Mills condition (and thus not bought at equilibrium) are priced by the hedonic price function when it is estimated. And they are priced according to Axiom 3. So although neither the continuous nor the discrete version of the Muth-Mills condition implies Axiom 3, the hedonic regressions used to test the standard model do imply it. This is due to the fact that distance from the CBD enters the right hand sides of the hedonic regressions in an additive manner, where the coefficient on distance from the CBD is the estimated commuting cost per unit of distance. The intuition for this restriction is exactly as Coulson suggests.

\footnotetext{
${ }^{4}$ In fact, Mills and Hamilton (1994, p. 110) requires some correction in this respect, since they refrain from using calculus in the text but nevertheless derive a discrete version of the result that uses an equality rather than inequalities.
} 
Axiom 3 is what sets urban economic hedonic models apart from hedonic models in other fields.

From the viewpoint of theory, Axiom 3 has real bite or is a refinement of hedonic equilibrium. If the bid rents of consumers cannot be ordered uniformly by steepness, then it is not necessarily the case that land or housing consumption increases with distance from the CBD in equilibrium. In that circumstance, identical houses or parcels can be purchased in equilibrium at different distances from the $\mathrm{CBD}$, and Axiom 3 is essentially a no-arbitrage condition. In the case where the theory implies increasing land or housing consumption with distance from the CBD, ${ }^{5}$ then Axiom 3 can be viewed as a refinement, in that if identical houses or parcels were purchased at different distances from the CBD, then in equilibrium Axiom 3 would be satisfied.

\section{The Result}

Theorem 1 Axioms 1, 2, and 3 together imply a contradiction. Thus, for any test of a hedonic housing model, at least one of the three axioms must be violated. If all three axioms are assumed (either explicitly or implicitly), then the model is internally inconsistent.

Proof: An almost trivial proof using calculations. Let the two different locations given in Axiom 1 be $x, y \in X$, let the two different housing types given in Axiom 1 be $h, i \in H$, and let the element of $G$ given in Axiom 1 be $g \in G$. Then Axiom 3 implies $P(h, x, g)=P(h, y, g)+t \cdot[y-x]$ and $P(i, x, g)=P(i, y, g)+t \cdot[y-x]$. Applying Axiom 2 to these two equations, $h \cdot p(x, g)=h \cdot p(y, g)+t \cdot[y-x]$ and $i \cdot p(x, g)=i \cdot p(y, g)+t \cdot[y-x]$. Note that the last two equations and $x \neq y$ imply that $p(x, g) \neq p(y, g)$. Combining the last two equations, one obtains that $h=i$, a contradiction. $\quad$ Q.E.D.

\footnotetext{
${ }^{5} \mathrm{~A}$ common set of sufficient conditions for bid rents to be ordered uniformly by steepness is that land or housing is a normal good, consumer preferences are identical, and consumers are endowed with no land but only a composite consumption good. Then in equilibrium, low endowment consumers live closest to the CBD while high endowment consumers live farthest from the CBD. In equilibrium, land consumption is increasing with distance from the CBD. These conditions are sufficient for existence of an equilibrium, but are much stronger than necessary.
} 


\section{The Take Home Lesson}

In this section, we will show that the "tests" of the monocentric city model are not tests at all. Even if the utility function and income of the identical consumers in the one type model are known to the observer, there is a continuum of models (equilibrium price systems) consistent with the observed housing purchases and rent density, only one of which is the standard model. If the income and utility function are unknown, there are huge numbers of models that are observationally equivalent to the standard model, having downward sloping rent and upward sloping land consumption. To make this idea more concrete, we present the argument in the abstract and then provide an example based on a common version of the standard model using log linear utility.

Note that the standard urban economic theory model satisfies Axiom 2 with $c \equiv 0$. Evidently, this model does not satisfy Axiom 3. However, it is easy to construct an equilibrium price system for such a model that does satisfy Axiom 3 but not Axiom 2. For simplicity, let's ignore $g$. Suppose that the equilibrium price of housing satisfying Axiom 2 is given by $p: X \rightarrow \Re$ (defining $P$ according to Axiom 2), and that the equilibrium housing function $h: X \rightarrow \Re$ is one-to-one and onto (for example, $h(0)=0$ and $d h / d x>k>0$ ). Denote the function $h^{-1}$ by $x(h)$. Define $\pi: H \times X \rightarrow \Re$ by $P(h, y, g)=$ $\pi(h, y) \equiv h \cdot p(x(h))+t \cdot[x(h)-y]$. Then the price system $p$ satisfies Axiom 2 but not Axiom 3. The price system $\pi$ satisfies Axiom 3 but not Axiom 2. The two price systems agree on the equilibrium bundles $h(x)$, but no price system satisfies both Axioms. Moreover, for any $\alpha \in(0,1)$, define the price system $P(h, x, g)=\pi^{\alpha}(h, y) \equiv \alpha h p(y)+(1-\alpha) \pi(h, y)$. Then $\pi^{\alpha}$ is also an equilibrium price system (with the same equilibrium bundles), but satisfies neither Axiom 2 nor Axiom 3.

Next we prove formally that if $p$ is an equilibrium price system, so is $\pi$ (with the same equilibrium bundles). To see this, let $u(h, z)$ be the utility function of all of the identical consumers, where $z$ is a non-negative number representing composite good consumption. If $w$ is a positive number representing income, the budget constraint is $w \geq t \cdot x+P(h, x)+z$. Now suppose that $[h(x), z(x), p(x)]$ is an equilibrium in the sense that $w \geq t \cdot x+h(x) \cdot p(x)+z(x) \forall x$ and for all $\left(h^{\prime}, z^{\prime}, x^{\prime}\right)$ with $w \geq t \cdot x^{\prime}+h^{\prime} \cdot p\left(x^{\prime}\right)+z^{\prime}, u(h(x), z(x)) \geq u\left(h^{\prime}, z^{\prime}\right)$. Suppose that $\exists\left(h^{\prime}, z^{\prime}\right)$ and $\exists x$ with $u\left(h^{\prime}, z^{\prime}\right)>u(h(x), z(x))$. Then $\forall x^{\prime}, t \cdot x^{\prime}+$ $\pi\left(h^{\prime}, x^{\prime}\right)+z^{\prime}=t \cdot x^{\prime}+h^{\prime} \cdot p\left(x\left(h^{\prime}\right)\right)+t \cdot\left[x\left(h^{\prime}\right)-x^{\prime}\right]+z^{\prime}=h^{\prime} \cdot p\left(x\left(h^{\prime}\right)\right)+t \cdot x\left(h^{\prime}\right)+z^{\prime}>w$. In fact, $\forall \alpha \in[0,1], P(h, x, g)=\pi^{\alpha}(h, y) \equiv \alpha h p(y)+(1-\alpha) \pi(h, y)$ is also an equilibrium price system (this follows easily from the fact that the equilibrium 
allocation is the same), and $\pi^{\alpha}$ satisfies neither Axiom 2 nor Axiom 3 when $\alpha \in(0,1)$.

Example 2 Take $u(h, z)=z+\ln (h)$, where the consumers are all identical and of measure $N$. For computational ease, consider a linear city so that the land available at each location is 1 . Calculations using first order conditions yield an equilibrium rent density of $p(x)=A e^{-x t}$, a standard exponential density, where $A=\frac{N t}{1-e^{-\overline{x t}}}$ and $\bar{x}$ is the extent of the city. Moreover, equilibrium housing consumption is $h(x)=A^{-1} e^{x t}$, so $x(h)=\ln (A h) / t$ and $\pi(h, y) \equiv$ $h \cdot p(x(h))+t \cdot[x(h)-y]=1+\ln (A h)-t \cdot y$, while $\pi^{\alpha}(h, y) \equiv \alpha h A e^{-y t}+$ $(1-\alpha)[1+\ln (A h)-t \cdot y]$. The price systems $p, \pi$, and $\pi^{\alpha}$ are equilibrium prices. The equilibrium allocations are the same for all of these equilibrium prices, though only $p$ is derived from the standard model and satisfies Axiom 2. The price system $\pi$ satisfies Axiom 3, while for any $\alpha \in(0,1), \pi^{\alpha}$ satisfies neither Axiom 2 nor Axiom 3.

\section{Conclusions}

Clearly what we have in mind is nihilism (as opposed to the hedonism of hedonic models). There is no price system that simultaneously satisfies two natural no arbitrage conditions, Axioms 2 and $3 .^{6}$ For each condition, there is one price system satisfying it and a continuum of price systems satisfying neither. These price systems generate the same equilibrium bundles, but price bundles not purchased in equilibrium differently. These models (with alternate price systems) are observationally equivalent, so the tests of the standard hedonic model cannot distinguish among them. The use of one particular price system out of a continuum represents an assumption that cannot be tested. ${ }^{7}$

The main advantage of price systems or models satisfying Axiom 2 is that it's possible that every equilibrium allocation will be efficient; see Berliant,

\footnotetext{
${ }^{6}$ It is amusing to note that most theorists prefer Axiom 2, and most empiricists prefer Axiom 3. Since the two axioms cannot be distinguished empirically, this must somehow be connected to matters of faith.

${ }^{7}$ Scotchmer (1986) is a related model. There, the hedonic price function is assumed linear and as a result is identified, but the preferences consistent with an equilibrium hedonic price function are not. As a consequence, the benefits of non-marginal changes in amenities ( $g$ in our notation) are not identified. In our model, the hedonic price function itself is not identified (outside of equilibrium values), so the benefits of non-marginal changes in amenities are unknown. This follows because it is unclear how such non-marginal changes will affect prices of or willingness to pay for locational goods via $c(g)$ or $P(h, x, g)$, as the only values of these functions that are known are those for equilibrium values of $g$.
} 
Papageorgiou and Wang (1990) for some counterexamples in small variations of the standard model. If one chooses a model and price system consistent with Axiom 3 instead of Axiom 2, then the nonlinear pricing in housing can generate more equilibria, perhaps with heterogeneous housing in each location. This would correspond closer to the data. On the other hand, such equilibrium allocations are unlikely to be efficient.

Coulson (1989) presents empirical evidence that Axiom 2, linear prices for housing at a given location, is violated. Evidently, this argues for acceptance of Axiom 3 in its place.

Let us turn next to a detailed discussion of Theorem 1 . One might think that a way to get around the problem we have presented is to estimate a hedonic model where commuting cost is not additively separable in price. However, this approach would deny the essential intuition of Axiom 3, that the prices of identical houses in different locations should be the same except for the difference in commuting cost. If a hedonic model is additively separable in commuting cost, then it likely violates Axiom 2.

The result can be extended in a number of ways. First, multiple city subcenters and different directions of commuting can be accommodated, as long as there are two locations from which consumers commute to the same subcenter. As Coulson (1991) notes, multiple incomes or types of consumers make no difference to Axiom 3 (or the other axioms). Notice that the construction of the hedonic regression itself, linear in distance from the CBD, implies Axiom 3, and thus leads to a contradiction. If we actually observe two pairs of identical houses $^{8}$ in different locations, then even without the specification of the hedonic regression model, we've got a serious problem, since Axiom 3 is satisfied (this is essentially the example given in the introduction). The latter idea can be extended to chains of pairs of identical houses, where the locations are the same for the second element of one pair and the first element of the next. If we get a non-trivial cycle of pairs (returning to the location where the chain starts), then there is a contradiction. The proof involves stringing together all of the equations implied by the chain. Non-linear commuting cost can be accommodated easily in the axioms and proof. Finally, the locational good need not be housing. One could replace all occurrences of "housing" in this paper by "land," where the differences in land quantities are simply different size parcels. The symbol $h$ would represent land consumption in that case.

There is yet another level of generalization of our result that is feasible;

\footnotetext{
${ }^{8} \mathrm{~A}$ convenient example is the "Chicago bungalow."
} 
it replaces the semiparametric regression in Axiom 2 with a non-parametric regression. Let $y>x$. Retain Axioms 1 and 3. These alone imply that $P(h, x, g)-P(h, y, g)=P(i, x, g)-P(i, y, g)$. In differential terms, this would imply that $\partial P(h, x, g) / \partial x \equiv \partial P(i, x, g) / \partial x$ (or its messy discrete alternative). Thus, $\partial^{2} P(h, x, g) / \partial x \partial h=0$ (or its messy discrete alternative). In place of Axiom 2, one can use the generalization $\partial^{2} P(h, x, g) / \partial h \partial x \neq 0$ or its messy discrete equivalent. (When Axioms 2 and 3 are satisfied, $\partial^{2} P(h, x, g) / \partial h \partial x=$ $-t$.) This yields a contradiction with a new, more general condition on the hedonic price function than no arbitrage. It is essentially a single crossing condition on $P$ ! This avenue of research clearly deserves further attention; supermodularity could play a key role.

There are two immediate implications of this analysis. First, it should be clear that when we test urban economic models, we are actually assuming more than the classical Muth-Mills condition. We are generally using Axiom 3 (stronger than the Muth-Mills condition). Second, if one attempts to tell a story about an auctioneer setting prices, then having Axiom 3 apply only to the levels of housing actually sold in equilibrium seems silly.

So where do we go from here? There are three obvious paths and one less obvious path. The first is to make commuting cost individual-specific. This path is likely to lead nowhere for two reasons. First, the framework presented here remains a special case, so the results still apply. Second, the empirical evidence (Deacon and Sonstelie (1985)) is that commuting cost is not heterogeneous.

The second path is to study imperfect competition models of the housing market. The hedonic regressions would have to be modified, for example to account for the number of firms in the industry. Again, the framework presented here will be a special case when firms have little or no market power, so this path does not seem promising either.

The third path is to allow commuting cost to depend on both distance from the CBD and on $h$. There are two problems with this idea. First, $h$ is a choice variable of each household. Does it make sense to have households choosing housing knowing that this choice affects commuting cost independent of distance from the CBD? This property seems unrealistic. Second, Axioms 2 and 3 imply that commuting cost must be proportional to $h$. So, for example, commuting cost at any location must vanish as $h$ tends to zero. Again, this seems unrealistic. 
The fourth and less obvious path ${ }^{9}$ is to allow nonlinearities of the hedonic price function $P(h, x, g)$ in both housing $h$ and in location $x$. For instance, one could use a nonparametric regression to test the hypothesis that $P$ is linear in $h$ or $x$, in other words, to see if Axioms 2 or 3 are satisfied. Thus, the data itself would speak to the issue of whether or not the no arbitrage conditions are satisfied. Of particular interest is whether or not Axiom 2 is consistent with the data, since this would be a real test of the standard model. ${ }^{10}$

\section{References}

[1] Berliant, M., Y.Y. Papageorgiou and P. Wang, 1990, On welfare theory and urban economics, Regional Science and Urban Economics 20, 245-261.

[2] Coulson, N.E., 1989, The empirical content of linearity-as-repackaging hypothesis, Journal of Urban Economics 25, 295-309.

[3] Coulson, N.E., 1991, Really useful tests of the monocentric model, Land Economics 67, 299-307.

[4] Deacon, R. and J. Sonstelie, 1985, Rationing by waiting and the value of time: Results from a natural experiment, Journal of Political Economy 93, 627-647.

[5] Ellickson, B., B. Grodal, S. Scotchmer and W. Zame, 2000, Clubs and the market, Econometrica 67, 1185-1217.

[6] Fujita, M., 1989, Urban Economic Theory (Cambridge University Press, Cambridge, UK).

[7] Kau, J.B. and C.F. Sirmans, 1979, Urban land value functions and the price elasticity of demand for housing, Journal of Urban Economics 6, $112-121$.

[8] Mas-Colell, A., 1975, A model of equilibrium with differentiated commodities, Journal of Mathematical Economics 2, 263-295.

\footnotetext{
${ }^{9}$ We are grateful to Nancy Wallace for this suggestion.

${ }^{10}$ Notice that the standard model predicts that housing or land consumption should be homogeneous at almost every location. If the standard model satisfying Axiom 2 is the null hypothesis, then under the null hypothesis, one should certainly never observe nonlinearities in the hedonic price function at a given location, both because housing is homogeneous (so only one value of $h$ should be observed) and because the pricing function is linear. Thus, it is certainly possible to reject the model.
} 
[9] McMillen, D.P., 1990, Consistent estimation of the urban land value function, Journal of Urban Economics 27, 285-293.

[10] McMillen, D.P., 2003, The return of centralization to Chicago: Using repeat sales to identify changes in house price distance gradients, Regional Science and Urban Economics 33, 287-304.

[11] Mills, E.S., 1969, The value of urban land, in The Quality of the Urban Environment, ed. by Harvey Perloff (Resources for the Future, Washington, DC) 231-253.

[12] Mills, E.S. and B.W. Hamilton, 1994, Urban Economics (Fifth Edition) (Harper Collins, NY, New York).

[13] Rosen, S., 1974, Hedonic prices and implicit markets: Product differentiation in pure competition, Journal of Political Economy 82, 34-55.

[14] Scotchmer, S., 1986, The short-run and long-run benefits of environmental improvements, Journal of Public Economics 30, 61-81.

[15] Yinger, J., 1979, Estimating the relationship between location and the price of housing, Journal of Regional Science 19, 271-289. 